\title{
Breastfeeding and Health Outcomes: A Narrative Review
}

\author{
Sahaya S Thresa
}

\begin{abstract}
Introduction: India is home to a large number of under-five deaths and underweight children in the world, which is mainly because of improper breastfeeding practices. Breastfeeding is one of the most effective ways to ensure child health and survival. Lack of exclusive breastfeeding during the first 6 months of life contributes to over a million avoidable child deaths each year.

Materials and methods: An extensive search of all materials related to the topic was carried out in PubMed, Medline, World Health Organization website, and Google Scholar search engines. Keywords used in the search included breastfeeding, colostrum, and infant. Overall, 30 articles were selected and analyzed.

Results: Parameters like educational status, socioeconomic status, occupation, mode of delivery, the place of delivery, and exclusive breastfeeding had a significant impact on the prevalence of exclusive breastfeeding.

Conclusion: The study participants had good knowledge about breastfeeding however, some gaps were observed with respect to their practices. The study concluded that the importance of increasing awareness among pregnant women about breastfeeding, and even gave impetus on the necessity of family support to aid mothers in showing adherence to the breastfeeding for the recommended duration.

Keywords: Breastfeeding, Colostrum, Infant.

Annals of SBV (2019): 10.5005/jp-journals-10085-8103
\end{abstract}

\section{INTRODUCTION}

Breastfeeding is a socially constructed practice, and its role is very essential in the formation of another individual, development of an effective immune system, brain function establishment, socialization, and advancing long-term health. ${ }^{1}$ Further, it has been observed that breastfed children have a much better intelligence quotient and even better school attendance. Moreover, by means of improvement in the child development, it will not only result in a reduction of the health expenses but will play a defining role in the economic gains for individual, their households as well as society. ${ }^{2}$ The probability of death among infants those were not breastfed exclusively are 15 times higher of developing pneumonia and 11 times due to diarrheal disease than exclusively breastfed children. ${ }^{3}$ The death rate is 14 times higher among those children who were not breastfed compared to exclusive breastfed (EBF) infants. ${ }^{4}$ Though it is broadly established that breast milk is good for the baby, it is also evidenced that adequate breastfeeding rates in early infancy are still too low. ${ }^{4}$ As per NFHS-4, of all the under-five deaths, undernutrition is the underlying cause of the estimated $31 \%$ of deaths in the rural area of Tamil Nadu, and the prevalence rate of early initiation of breastfeeding is only $54.2 \%$, and exclusive breastfeeding is only $48.7 \% .^{5}$

Thus, awareness creation about this deadly disease and promotion of breastfeeding becomes the need of the hour, which can be achieved with the help of information, education, and communication activities. ${ }^{6}$ Many health campaigns focused on breastfeeding behavior by proper education of all mothers would contribute to reducing the burden of breast cancer. ${ }^{7}$

\section{Materials and Methods}

An exhaustive search for all materials related to the topic was made in different search engines, including PubMed, Medline, World Health Organization website and Google Scholar, and Government websites for a duration of 15 days. Relevant policy documents, systematic reviews and meta-analysis, case-control studies, cohort
Department of Community Medicine, Sri Sathya Sai Medical College and Research Institute, Chennai, Tamil Nadu, India

Corresponding Author: Sahaya S Thresa, Department of Community Medicine, Sri Sathya Sai Medical College and Research Institute, Chennai, Tamil Nadu, India, Phone: +91 8056025885, e-mail: sonathresa@yahoo.com

How to cite this article:Thresa SS. Breastfeeding and Health Outcomes: A Narrative Review. Ann SBV 2019;8(1):9-12.

Source of support: Nil

Conflict of interest: None

studies, cross-sectional surveys, technical publication series, and research articles published in the period from 1995 to 2019 were included in the review. A total of 47 studies were identified. Seventeen studies were excluded because of the unavailability of the full version of the article. Overall, 30 articles were selected based upon the suitability with the current review objectives and analyzed. Keywords used in the search include breastfeeding, infant, colostrum, and exclusive breastfeeding.

\section{Global Strategy for Infant and Young Child Feeding}

Millennium Development Goal-4 aims to reduce the mortality rate in under-five children by $2 / 3$ by 2015 , which cannot be achieved without reducing the neonatal deaths that alone accounts for almost $40 \%$ of under-five deaths. ${ }^{8}$ It has been estimated that 4 million neonatal deaths are preventable with causes like pneumonia, sepsis, meningitis being the major reasons for infant and neonatal deaths in developing countries. ${ }^{9}$ Early initiation and promotion of exclusive breastfeeding contribute to a large extent in minimizing both neonatal and infant mortality, to eventually accomplish the Millennium Development Goals (MDGs). ${ }^{9}$ Indeed, initiating breastfeeding earlier and exclusive breastfeeding for the initial 6 months of life will play a crucial role in ensuring the young infants are exposed to the best possible start for a healthy life. ${ }^{10}$ Worldwide, it is generally recommended that the newborn child 
must only be breastfed for the initial 6 months of life to obtain ideal development, improvement, and well-being. ${ }^{10}$ From there on, to meet their required nourishment, babies ought to get nutritiously sufficient and safe reciprocal substances while breastfeeding proceeds up to 2 years old. ${ }^{10}$

\section{National Guidelines for Infant and Young Child Feeding}

- WHO/UNICEF has emphasized that 270 days in utero and the first 1,000 days of life after birth (initial 2 years of age), as the crucial window for IYCN (infant and young child nutrition) interventions. $^{11}$

- Gold standard feeding options should be offered for babies by ensuring timely counseling of the mothers and caregivers. ${ }^{11}$

- Predelivery counseling, separately, or in gatherings should be organized by the local maternity facilities for all the mothers and it should be centered on the advantages of breastfeeding and the demerits of formula feeding. ${ }^{11}$

- Skin-to-skin contact should be started within 5 minutes of birth in every single typical infant (counting those by cesarean section). The "Breast Crawl" strategy can also be used for the early initiation of breastfeeding in an hour of birth. The newborn ought to be kept warm by advancing Kangaroo Mother Care and elevating neighborhood practices to keep the room warm if the infant is low birth weight. ${ }^{11}$

- Colostrum should be nourished to newborn as it has a high density of defensive immune globulins and cells, hence it must not be discarded. No prelacteal liquid ought to be given to the newborn. ${ }^{11}$

- Babies should be breastfed no less than 8 to 10 times a day. ${ }^{11}$

- Exclusive breastfeeding is to be polished from birth until the end of 6 months. Though breastfeeding has to be continued for a period of 2 years and beyond, optimal complementary feeding can be initiated after the completion of 6 months of age. ${ }^{11}$

- Hirkani's rooms might be considered as work areas, especially for breastfeeding mothers who can drain milk and store in an icebox amid working hours."

- During every antenatal and postnatal visit, the harms of container and formula feeding should be explained to the mother, and it can only be practiced if medically indicated. ${ }^{11}$

- WHO recommends re-lactation through supplemental suckling technique (SST). The trickle and drop technique supports the newborn child's enthusiasm for suckling at the breast. ${ }^{11}$

- Nursing in public (NIP)_mothers should be comfortable enough to nurse the baby in public. ${ }^{11}$

\section{Advantages of Breastfeeding}

\section{Benefits to the Child}

Benefits of breastfeeding are as follows, breast milk is always safe, clean, hygiene, and cheap, and it protects the baby from infectious diseases like diarrhea, pneumonia. ${ }^{8}$ Breast milk is the best natural food for infants. ${ }^{8}$ It is the only food available 24 hours without any special preparation. ${ }^{8}$ Breastfeeding helps to develop better intelligence quotients and visual acuity because of the presence of special fatty acids in breast milk and helps in preventing babies from developing obesity. ${ }^{12}$ Breastfeeding is the natural way of nursing the child, and it helps to establish a solid bond between the mother and the infant. ${ }^{13}$ Breastfeeding gives a lifelong impact on psychological development and emotional security and affection. ${ }^{13}$

Exclusive breastfeeding is the base for child health and survival as it provides adequate nutrients for a child's growth and advancement. ${ }^{13}$ It is microbiologically sterile with less chance of contamination. Breast milk serves as a child's first vaccination, prevents respiratory infections, diarrheal disease, and other possibly life-threatening illness. ${ }^{13}$ Exposure to infection is more common in developing countries where human milk is protective and safe food for infants. ${ }^{13}$ Breast milk has appropriate anti-infective functions macrophages, lysozyme, secretory lgA, lymphocytes, and lactoferrin are the antimicrobial factors in human milk, which no other milk has. ${ }^{14}$ Certain noncommunicable diseases like obesity can be prevented by exclusively breastfeeding children. ${ }^{13}$ Breastfed child has 8 points higher intelligent quotient due to the presence of taurine, docosahexaenoic acid, choline. In short, breastfeeding is an incomparable way of delivering the ideal food for the development of infants and well-being. ${ }^{12}$

\section{Benefits to the Mother}

Breastfeeding induces oxytocin release that promotes bonding between the mother and the baby. ${ }^{8}$ Breastfeeding is a natural contraceptive method, which delays the next pregnancy. It also helps mothers to gain prepregnancy weight, and protects from ovarian and breast cancers. ${ }^{8}$ Breastfeeding for a longer time also benefits well-being and health of mothers. ${ }^{13}$ It helps in space pregnancies-exclusive breastfeeding of infants below 6 months has a hormonal outcome that habitually stimulates a lack of menstruation and also lowers the risk of ovarian and breast cancer. ${ }^{13}$ The EBF is a natural (though not fail-safe) known as lactation amenorrhea method of birth control method. ${ }^{13}$ Also, it is an essential element of the reproductive procedure with significant implications for the well-being of mothers. ${ }^{10}$

\section{Factors Influencing Breastfeeding}

An extensive variety of factors have been identified in various epidemiological studies, which can affect the practice of breastfeeding, namely

- Bhatt Shwetal et al. reported that the percentage of mothers starting breastfeeding earlier was highest among literate mothers than illiterate mothers. ${ }^{4}$

- Kumar et al. reported that the reason for low prevalence could be a lack of support from family members, absence of advice from health care staffs in the period of ANC visits and deliveries conducted in institutions that are not babyfriendly. ${ }^{14}$

- Patel et al., in his study of the indicators and determinants of poor infant feeding practices reported that factors like educational status of the mother, antenatal visits, antenatal advice on infant feeding and mass media had a significant impact on the exclusive breastfeeding practice. ${ }^{15}$ Risk factors for bottle-feeding were like cesarean delivery, absence of postnatal examination, higher household wealth index. ${ }^{15}$

- Tiwari et al. reported the significance of the health education of both the mother and the family members in ideal breastfeeding practices. ${ }^{16}$

- Normal vaginal delivery and the infant's present weight are the positive predictors of giving colostrum. ${ }^{17}$

- Britton et al. had emphasized the overall support from the aspect of the health care providers and family to the nursing mothers in infant feeding practices. ${ }^{18}$

- Saied et al., the study showed that the occupation of mothers $73.5 \%$ is one of the common barriers for initiation of breastfeeding. ${ }^{19}$ 
- Kishore and Joshi, noticed the low prevalence of breastfeeding was mostly due to a lack of support from family members, deliveries conducted in non-baby-friendly hospitals, and lack of advice from health staff from family members. ${ }^{20}$

\section{Early Initiation of Breastfeeding}

Colostrum is the first milk from the mother to the newborn; ideally, the baby should be breastfed as early as possible and most probably within an hour of birth. ${ }^{13}$ The early initiation of breastfeeding helps the infants to learn to suck soon after birth when the baby is active. This early suckling by infants helps in milk formation and early secretion of breast milk. The newborn should be kept close to the mother for frequent feeding and also to maintain the temperature of the babies. Early initiation of breastfeeding will also help in better milk flow and secretion of milk. Initiation of breastfeeding inside the principal hour of life by skin-to-skin contact leads to strong bonding amongst mother and baby quickly after birth. ${ }^{13}$

\section{Value of Colostrum}

The milk secreted in the initial few days of life after the birth of the baby is called colostrum. It is sticky and yellowish in nature, rich in protein and vitamin A. ${ }^{11}$ It is highly nutritious and anti-infective, also the first immunization given to the child by her/his mother after birth. It helps developing nutrients and anti-infectious substances in the infant's body during the first few weeks of life. It has less fat and carbohydrate lactose as compared to matured milk. Due to the different color and consistency of the colostrums, the first milk was considered as dirty and indigestible by some mothers. Adequate education to be given to the mothers and the community about the importance of colostrum would help to ensure that colostrum is fed to the child but not wasted. Mothers may develop engorgement of the breast due to late initiation of breastfeeding. ${ }^{11}$

\section{Colostrum and Initiation of Breastfeeding}

Adequate education about the importance of colostrum to be given to the mother in the community to ensure that colostrum is to be fed to the child but not wasted. In India, $15.8 \%$ of the infants are begun with breastfeeding inside 1 hour of birth and just $37.1 \%$ within a day after birth. ${ }^{11}$ Vijayalakshmi et al. reported that most of the mothers knew the fact that colostrum is the first breast milk and plays a crucial role in maintaining the immunity of the child. ${ }^{21}$ Sachdev and Mehrota et al. reported that exclusive breastfeeding in early infancy like colostrum as the first feed. ${ }^{22}$ Ratan and Mukherjee et al. reported that $64 \%$ of mothers had a good opinion about colostrum. ${ }^{23}$ Appropriate knowledge about breastfeeding initiation time among vaginal delivery and cesarean mothers' were $76.9 \%$ and $65.2 \%$, respectively. ${ }^{24}$ Harnagle et al., in his study, disclosed that $63.6 \%$ of the women breastfed their newborn within 6 hours of birth. ${ }^{25}$ Paudel et al. reported that avoiding prelacteal feeding was considered to be good practice, and nearly ( $>4 / 5$ th) of the mothers had practiced avoiding prelacteal feeds. ${ }^{26}$

\section{Exclusive Breastfeeding}

- Prelacteal feeds are often given to a newborn fearing that the newborn may be hungry or may become dehydrated. ${ }^{27}$ Any food provided to a newborn before the initiation of mother's breastfeeding is considered to be a prelacteal feed. The type of prelacteal feeds depends on culture. It may include ghee (refined butter), honey, sugar, sugar juice, unboiled cow/goat milk, etc. ${ }^{28}$ Prelacteal feeds interferes with prolactin production and sucking and undermines the mother's confidence in her ability to breastfeed. ${ }^{29}$ Meshram et al., in his study, indicated that infants who received prelacteal feeding were significantly more prone to be stunted compared to those who were exclusively breastfed. ${ }^{30}$

- Sarmila Mallik et al. reported that $90 \%$ of mothers knew about the initiation of breastfeeding in 24 hours and proper knowledge about breastfeeding initiation time in vaginal delivery and cesarean mothers were $76.9 \%$ and $65.2 \%$, respectively. ${ }^{31}$

- Kommula et al., in her study revealed that only $36.3 \%$ of the participants knew about time of initiation after birth and $65.2 \%$ were aware of the value of feeding colostrum, correct duration of exclusive breastfeeding was about $47.4 \%$, nearly $63.7 \%$ had knowledge the benefits to the child due to breastfeeding, and very few know the benefits to the mother by breastfeeding the babies. ${ }^{32}$

- Dhammika et al., in her study, mentioned that $100 \%$ of the mothers had knowledge that only feeding breast milk for babies was known as exclusive breastfeeding and even had knowledge about the duration of EBF was 6 months. ${ }^{33}$

- Hiregoudar et al. reported that exclusive breastfeeding practice was seen significantly amid literate mothers, and mothers were informed about breastfeeding are those who delivered in a hospital. ${ }^{34}$

\section{CONCLUSION}

Antenatal counseling and health education were given in the government health facility, particularly in the primary health care level, which had a significant impact on the practices of exclusive breastfeeding among rural mothers. Only very few proportions of mothers were of the opinion that colostrum should be discarded owing to its infectious nature or because of being not healthy for babies. Breastfeeding initiation earlier with colostrum within the first hour of life has a significant impact on the future practice of exclusive breastfeeding. Further, it was observed that the introduction of complementary feeding at an appropriate time should be strictly practiced, as it has a significant effect on future practices. One to one counseling or health education given to the rural mothers by the health workers during the antenatal period, delivery and in the postnatal follow-up period, particularly during immunizations resulted in a significant influence on the practice of exclusive breastfeeding, and even prelacteal feeds to be avoided to undermines the mother's ability to breastfeed her child.

\section{References}

1. Damstra KM, Improving breastfeeding knowledge, self-efficacy and intent through aprenatal education program, 2012, Available from http://scholarworks.gvsu.edu/cgi/viewcontent.cgi?article $=1003 \&$ co ntext=dissertations [Last accessed on 2019 May 20].

2. World Health Organization. WHO Global Targets 2025. Available from www.who.int/nutrition/global-target-2025 [Last accessed on 2019 May 20].

3. UNICEF India. Nutrition - Latest stories Unicef India - 2014, 2014. Available from www.unicef.org/india/nutrition [Last accessed on 2019 May 12].

4. Shwetal B, Pooja P, Neha K, Amit D, Rahul P. Knowledge, attitude and practices of postnatal mothers for early initiation of breastfeeding in obstetric ward of tertiary carehospital of adadora city. Nat J Comm Med 2012;3(2):305-309.

5. Ministry of Health and Family Welfare. National Family Health Survey-4 2015-2016. State factsheet Tamil Nadu. Available from http:// 
rchiips.org/nfhs/pdf/NFHS4/TN_FactSheet.pdf [Last accessed on 2019 May 13].

6. Gajalakshmi V, Mathew A, Brennan P, Rajan B, Kanimozhi VC, Mathews $A$, et al. Breastfeeding and breast cancer risk in India: a multicenter case-control study. Int J Cancer 2009;125(3):662-665. DOI: 10.1002/ ijc.24429.

7. WHO, UNICEF. Global strategy for infant and young child feeding, World Health Organization, 2002. Available from www.who.int/childadolescent health/NUTRITION/global-strategy.htm [Last accessed on 2019 May 20].

8. Radhakrishnan S, Balamurga SS. Prevalence of exclusive breastfeeding practices among a. rural women in Tamil Nadu. Int J Health Allied Sci 2012;1(2):64-67. DOI: 10.4103/2278-344X.101667.

9. United Nations. Millennium Development Goals. Available from www. un.org/millenniumgoals [Last accessed on 2019 May 11].

10. WHO/Exclusive breastfeeding-World Health Organization. Available from www.who.int/nutrition/topics/exclusive_breastfeeding/en/ [Last accessed on 2019 May 13].

11. National Guidelines of Infant and Young Child Feeding, 2004. Available from http://wcd.nic.in/sites/default/files/nationalguidelines.pdf [Last accessed on 2019 May 19].

12. American Academy of Paediatrics Policy Statement. Breastfeeding and use of human milk. Paediatrics 2005;115:496-506.

13. Park K. Preventive medicine in obstetrics, paediatrics and geriatrics. In: Park K, ed. Text book of Preventive and Social Medicine, 24rd ed., Jabalpur: Banarsidas Bhanot Publishers; 2017. pp. 573-576.

14. Kumar P, Aggarwal AK. Breastfeeding Knowledge and practices amongst mothers in rural population of North India: community based study. J Trop Pediatr 2009;55(3):183-188. DOI: 10.1093/tropej/fmn110.

15. Patel A, Badhoniya N, Khadse S, Senarath U, Agho KE, Dibley MJ. Infant and young child feeding indicators and determinants of poor feeding practices in India: a review of National Family Health Survey data. Food Nutrition Bull 2010;31(2):314-333. DOI: 10.1177/156482651003100221.

16. Tiwari R, Mahajan PC, Lahariya C. Determinants of exclusive breastfeeding in urban slums: a community based study. J Trop Pediatr 2009;55(1):49-54. DOI: 10.1093/tropej/fmn037.

17. Eckhardt CL, Lutz T, Karanja N, Jobe JB, Maupome G, Ritenbaugh C. Knowledge, attitudes and beliefs that may influence infant feeding practices in American Indian Mothers. J Acad Nutr Diet 2014;114(10):1587-1593. DOI: 10.1016/j.jand.2014.04.021.

18. Britton C, McCormick FM, Renfrew MJ. Support for breastfeeding mothers - Cochrane Database Of Systematic Reviews; 2006.

19. Saied H, Mohamed A, Suliman A, Anazi WA. Breastfeeding knowledge, attitude and barriers among Saudi women in Riyadh. J Natural Sci Res 2013;12(3):1-11.

20. Kishore MS, Joshi PC, Angdembe MR, Das SK, Ahmed S, Faruque AS, et al. Prevalence of exclusive breastfeeding and associated factors among mothers in rural Bangladesh: a cross-sectional study. Int Breastfeed J 2014;9:7. DOI: 10.1186/1746-4358-9-7.

21. Vijayalakshmi P, Susheela T, Mythili D. Knowledge, attitudes, and breast feeding practices of postnatal mothers: a cross sectional survey. Int J Health Sci 2015;9(4):364-374. DOI: 10.12816/0031226.

22. Sachdev HP, Mehrota S. Predictors of exclusive breastfeeding in early infancy. Operational implications. Ind Pediatr 1995;32(12):1287-1296.

23. Ratan D, Mukherjee A. Knowledge, attitude and practices of infant feeding practices among rural women in eastern India. J Evolution Med Dental Sci 2014;3(2):506-512. DOI: 10.14260/jemds/2014/1857.

24. Patro S, Nanda S, Sahu R. Infant feeding practices of Paroja: A tribal community of Orissa. Stud Home Community Sci 2012;6(1):21-25. DOI: 10.1080/09737189.2012.11885352.

25. Harnagle R, Chawla PS. A study of knowledge, attitude and practices (kap) of lactating mothers on breast feeding, weaning immunization and dietary practices at Jabalpur cantonment, India. Int J Curr Microbiol App Sci 2013;2(11):393-403.

26. Paudel DP, Giri S. Breast feeding practices and associated factors in Bhaktapur District of Nepal: a community based cross-sectional study among lactating mothers. J Sci Soc 2014;41(2):108-113. DOI: 10.4103/0974-5009.132851.

27. Sathish K, Vallabh B. Prelacteal feeding practices in a rural community. Indian Med Gaz 2011; 337-341.

28. Laroia N, Sharma D. The religious and cultural bases for breastfeeding practices among the Hindus. Breastfeed Med 2006;1(2):94-98. DOl: 10.1089/bfm.2006.1.94.

29. Jayashree M. Lactation failure-myth or reality? J OBGY India 1999;49(4):78-82.

30. Meshram I, Laxmaiah A, Venkaiah K, Brahmam GNV. Impact of feeding and breastfeeding practices on the nutritional status of infants in a district of Andhra Pradesh, India. Natl Med J India 2012;25(4): 201-206.

31. Mallik S, Dasgupta U, Naskar S, Sengupta D, Choudary K, Bhattacharya SK. Knowledge of breast feeding and timely initiation of it amongst postnatal mothers: an experience from a baby friendly teaching hospitals of a metropolitan city. IOSR J Dent Med Sci 2013;4(1):25-30. DOI: 10.9790/0853-0412530.

32. Kommula ALDS, Kommula VM. Knowledge, attitude and practices of breast feeding among mothers in a slum area of Amalapuram, East Godavari District, Andhra Pradesh. Nat J Med dental Res 2014;2(3):15-17.

33. Dhammika BLK, Gunawardena NS. Knowledge, practices and concerns regarding exclusive breastfeeding for six months among mothers of infants in a suburban setting in Sri Lanka. Sri Lanka J Child Health 2012;41(1):9-14. DOI: 10.4038/sljch.v41i1.4130.

34. Hiregoudar V, Hemagiri K, Goud TG. Breast feeding practices in Bellary, Karnataka: a hospital based study. J Pub Health Med Res 2013;1(2):76-80. 